\title{
Pharmaceutical Intervention for Adverse Events Improves Quality of Life in Patients With Cancer Undergoing Outpatient Chemotherapy
}

Hironori Fujii ( $\sim$ h_fujii@gifu-u.ac.jp )

Gifu Daigaku Igakubu Fuzoku Byoin https://orcid.org/0000-0003-0944-7290

Yukino Ueda

Gifu Yakka Daigaku

Chiemi Hirose

Gifu Daigaku Igakubu Fuzoku Byoin

Koichi Ohata

Gifu Daigaku Igakubu Fuzoku Byoin

Kumiko Sekiya

Gifu Daigaku Igakubu Fuzoku Byoin

Mika Kitahora

Gifu Daigaku Igakubu Fuzoku Byoin

Shiori Sadaka

Gifu Daigaku Igakubu Fuzoku Byoin

Senri Yamamoto

Gifu Daigaku Igakubu Fuzoku Byoin

Daichi Watanabe

Gifu Daigaku Igakubu Fuzoku Byoin

Hiroko Kato-Hayashi

Gifu Daigaku Igakubu Fuzoku Byoin

Hirotoshi lihara

Gifu Daigaku Igakubu Fuzoku Byoin

Ryo Kobayashi

Gifu Daigaku Igakubu Fuzoku Byoin

Miho Kaburaki

Gifu Yakka Daigaku

Nobuhisa Matsuhashi

Gifu Daigaku Igakubu Daigakuin Igakukei Kenkyuka

Takao Takahashi

Gifu Daigaku Igakubu Daigakuin Igakukei Kenkyuka

Akitaka Makiyama

Gifu Daigaku Igakubu Daigakuin Igakukei Kenkyuka

Kazuhiro Yoshida

Gifu Daigaku Igakubu Daigakuin Igakukei Kenkyuka

Hideki Hayashi

Gifu Yakka Daigaku

Akio Suzuki

Gifu Daigaku Igakubu Fuzoku Byoin 


\section{Research Article}

Keywords: Quality of life, Pharmaceutical intervention, Adverse events related outpatient cancer chemotherapy, EuroQol 5 Dimension5 Level (EQ-5D-5L), retrospective observational study

Posted Date: December 2nd, 2021

DOI: https://doi.org/10.21203/rs.3.rs-974568/v1

License: @ (i) This work is licensed under a Creative Commons Attribution 4.0 International License. Read Full License

Version of Record: A version of this preprint was published at Journal of Pharmaceutical Health Care and Sciences on March 2nd, 2022. See the published version at https://doi.org/10.1186/s40780-022-00239-w. 


\section{Abstract}

\section{Purpose}

The effect of pharmaceutical intervention to treat adverse events on quality of life (QOL) in outpatients receiving cancer chemotherapy is unclear. We investigated whether pharmaceutical intervention provided by pharmacists in collaboration with physicians improves QOL with outpatient cancer chemotherapy.

\section{Methods}

We conducted a single-center retrospective descriptive study of pharmaceutical intervention for patients receiving outpatient cancer chemotherapy at Gifu University Hospital between September 2017 and July 2020. We assessed patient QOL using the Japanese version of the EuroQol 5 Dimension5 Level (EQ-5D-5L). Adverse events were graded using the Common Terminology Criteria for Adverse Events (CTCAE) version 4.0. We compared the EQ-5D-5L utility value and incidence of grade 2 or higher adverse events before and after pharmaceutical intervention.

\section{Results}

Our analysis included 151 patients who underwent 210 chemotherapy cycles. Pharmaceutical intervention significantly improved patients' EQ-5D-5L utility values from 0.8197 to $0.8603(P<0.01)$. EQ-5D-5L utility values were significantly improved after pharmaceutical intervention for nausea and vomiting (pre-intervention 0.8145, post-intervention 0.8603, $P=0.016$ ), peripheral neuropathy (pre-intervention 0.7798 , post-intervention $0.7988, P=0.032$ ) and pain (pre-intervention 0.7625 , postintervention $0.8197, P=0.035$ ). Although not statistically significant, the incidence of grade 2 or higher adverse events, including nausea and vomiting, dermopathy, pain, oral mucositis, diarrhea and dysgeusia, tended to be lower post-intervention than pre-intervention.

\section{Conclusions}

Pharmaceutical intervention by pharmacists in collaboration with physicians may improve QOL in patients undergoing outpatient cancer chemotherapy.

\section{Introduction}

Cancer is a leading cause of death, and cancer incidence is increasing globally, with a corresponding increase in the number of patients receiving cancer chemotherapy. [1, 2]. Historically, anticancer drug therapy was performed only in hospital settings, but advances in the development of supportive therapy and modifications to the healthcare environment to reduce medical costs have facilitated broad-scale outpatient cancer chemotherapy [3, 4]. Although quality of life (QOL) is higher in patients receiving outpatient cancer chemotherapy than in those treated in hospital [5], the decreased access to medical management places these patients at risk of worse outcomes for several adverse events associated with anticancer drug therapy $[6,7]$.

We previously reported a significant correlation between adverse events, such as peripheral neuropathy, malaise and edema of the limbs, and decreased QOL, regardless of type of cancer or anticancer drug [8]. Similarly, Blanca Prieto-Callejero et al. reported that adverse events, such as nausea, dysgeusia, peripheral neuropathy and loss of appetite have a negative impact on QOL [9]. Iwamoto et al. reported severe mucositis/stomatitis affected health-related QOL in patients treated with cetuximab plus standard chemotherapy for advanced/metastatic colorectal cancer [10]. Moreover, Mol et al. found that patients with advancedstage lung cancer who experienced strong negative feelings regarding the side effects of chemotherapy also showed decreased QOL, and recommended that physicians facilitate vigorous management of low-grade adverse events to improve QOL [11]. These reports highlight the necessity of reducing adverse events to enhance QOL. In turn, medical staff, including pharmacists, should therefore conduct monitoring and management for patients receiving outpatient-cancer chemotherapy. Pharmacists have the important role of selecting the appropriate drug therapy and contributing to adverse event reduction and supportive care by communicating with patients receiving cancer chemotherapy through patient interviews [12-14]. Pharmaceutical interventions have been shown to improve QOL in outpatients receiving cancer chemotherapy [15-17]. Although some studies 
have reported that patient education conducted by pharmacists had a positive impact, the effect of pharmaceutical intervention provided in collaboration with physicians to manage adverse events has not been evaluated.

Here, we investigated whether pharmaceutical intervention provided by pharmacists in collaboration with physicians improved QOL in outpatient cancer chemotherapy.

\section{Methods}

\section{Study design}

This retrospective observational study was conducted at Gifu University Hospital. Patients who underwent outpatient cancer chemotherapy between September 2017 and July 2020 were enrolled. We extracted utility values of QOL, adverse events, pharmaceutical intervention for adverse events, chemotherapy regimens, and other patient demographics from electronic medical records. We included patients who underwent pharmaceutical intervention by pharmacists in collaboration with physicians for adverse events, and excluded patients for whom pre- or post-intervention assessment of QOL was missing or incomplete.

\section{Assessment of QOL}

We used EuroQol 5 Dimension 5 Level (EQ-5D-5L) to assess health-related QOL in patients undergoing outpatient cancer chemotherapy. The EQ-5D-5L questionnaire is a generic health status measure developed by the EuroQol group [18] to calculate quality-adjusted life years [19]. We used the Japanese version of the EQ-5D-5L, which was developed by Shiroiwa et al. in 2015 [20].

The questionnaire consists of five dimensions: mobility, self-care, usual activities, pain/discomfort, and anxiety/depression. Each dimension has 5 levels: level 1, no problem; level 2, slight problem; level 3, moderate problem; level 4, severe problem; level 5 , unable or extreme [18]. A utility value ranging from 0 to 1 is calculated from the EQ-5D-5L, in which 0 indicates death and 1 indicates full health [21].

Patients who underwent outpatient cancer chemotherapy were asked to answer the EQ-5D-5L questions after each cycle.

\section{Assessment of adverse events}

In the outpatient chemotherapy clinic, physicians, pharmacists and nurses collaborated to assess adverse events, such as nausea and vomiting, diarrhea, oral mucositis, dysgeusia, peripheral neuropathy, pain, malaise, alopecia, and skin disorders. The severity of each adverse event was graded according to the Common Terminology Criteria for Adverse Events (CTCAE) version 4.0 [22]. Physicians and pharmacists provided pharmaceutical intervention based on clinical practice guidelines to treat moderate or severe adverse events occurring during cancer treatment, and the effect of the intervention on the particular adverse event was assessed at the next consultation.

\section{Effect of pharmaceutical care on adverse events}

We evaluated changes in QOL in patients who developed adverse events at two points, namely the onset of the adverse event (pre-intervention) and after pharmaceutical intervention for the adverse events (post-intervention).

\section{Statistical analysis}

We used IBM SPSS version 22 (IBM Japan Ltd, Tokyo, Japan) to analyze data. P-values $<0.05$ were considered statistically significant. We summarized continuous variables using medians with 25 th and 75 th percentiles and categorical variables using frequencies and percentages.

The Wilcoxon signed-rank test was conducted to assess the effect of pharmaceutical interventions on QOL in patients experiencing adverse events. We compared the incidence of grade 2 or higher adverse events between pre- and post-intervention 
using McNemar's test.

\section{Results}

\section{Patients}

Among patients who received pharmaceutical intervention for adverse events between September 2017 and July 2020, QOL was measured using EQ-5D-5L in 302 cases. Among these, we excluded 84 cases that were exacerbated by adverse events unrelated to the intended intervention and 8 cases that did not have complete QOL data. We included 210 interventions (151 patients) in the analysis (Figure 1). Patient characteristics are summarized in Table 1. The EQ-5D-5L questionnaire was obtained from 151 patients who received pharmaceutical intervention for adverse events. The most common type of cancer was breast cancer (27.8\%), followed by colorectal (26.5\%), pancreatic (13.2\%), gastric (11.3\%), head and neck (6.0\%), and lung (6.0\%). The most common type of regimen was oxaliplatin-based chemotherapy (21.2\%), followed by paclitaxel/nanoparticle albumin-bound paclitaxel-based chemotherapy (17.9\%), anthracyclines + cyclophosphamide (11.3\%), gemcitabine + nanoparticle albumin-bound paclitaxel (7.9\%), and irinotecan-based chemotherapy (7.3\%). 


\begin{tabular}{|c|c|c|}
\hline Number of patients (male/female) & 151 & $(63 / 88)$ \\
\hline Age, median (min-max) & 60 & $(28-82)$ \\
\hline Number of chemotherapy courses & 210 & \\
\hline \multicolumn{3}{|l|}{ Cancer } \\
\hline Breast cancer & 42 & $27.8 \%$ \\
\hline Colorectal cancer & 40 & $26.5 \%$ \\
\hline Pancreatic cancer & 20 & $13.2 \%$ \\
\hline Gastric cancer & 17 & $11.3 \%$ \\
\hline Head and neck cancer & 9 & $6.0 \%$ \\
\hline Lung cancer & 9 & $6.0 \%$ \\
\hline Ovarian cancer/cervical cancer/uterine cancer & 5 & $3.3 \%$ \\
\hline Biliary tract cancer & 4 & $2.6 \%$ \\
\hline Angiosarcoma & 1 & $0.7 \%$ \\
\hline Esophageal cancer & 1 & $0.7 \%$ \\
\hline Malignant lymphoma & 1 & $0.7 \%$ \\
\hline Malignant pleural mesothelioma & 1 & $0.7 \%$ \\
\hline Peritoneal cancer & 1 & $0.7 \%$ \\
\hline \multicolumn{3}{|l|}{ Regimen } \\
\hline L-OHP+fluoropyrimidines \pm Bmab/Pmab/Tmab & 32 & $21.2 \%$ \\
\hline PTX/Nab-PTX \pm Tmab/ramucirumab/Bmab/Cmab & 27 & $17.9 \%$ \\
\hline Anthracyclines+cyclophosphamide & 17 & $11.3 \%$ \\
\hline GEM+Nab-PTX & 12 & $7.9 \%$ \\
\hline CPT-11 \pm fluoropyrimidines \pm Bmab/aflibercept/ramucirumab/Pmab & 11 & $7.3 \%$ \\
\hline DOC/GEM/EPI/VNR/NabPTX+PER+Tmab & 10 & $6.6 \%$ \\
\hline FOLFIRINOX/FOLFOXIRI+Bmab & 8 & $5.3 \%$ \\
\hline $\mathrm{CBDCA} \pm \mathrm{PEM} \pm \mathrm{Bmab} \pm \mathrm{Cmab}$ & 7 & $4.6 \%$ \\
\hline $\mathrm{GEM} \pm \mathrm{S}-1 \pm \mathrm{CDDP}$ & 7 & $4.6 \%$ \\
\hline Pembrolizumab/nivolumab/durvalmab/atezolizumab & 6 & $4.0 \%$ \\
\hline TAS102+Bmab & 4 & $2.6 \%$ \\
\hline DOC/LipoDOX $\pm B m a b$ & 3 & $2.0 \%$ \\
\hline Maintenance chemotherapy (Tmab/rituximab) & 2 & $1.3 \%$ \\
\hline
\end{tabular}

L-OHP, oxaliplatin; Bmab, bevacizumab; Pmab, panitumumab; Tmab, trastuzumab; PTX, paclitaxel; Nab-PTX, nanoparticle albumin-bound paclitaxel; Cmab, cetuximab; GEM, gemcitabine; CPT-11, irinotecan; DOC, docetaxel; EPI, epirubicin; VNR, vinorelbine; PER, pertuzumab; FOLFIRINOX/FOLFOXIRI, L-OHP+CPT-11+5-FU; CBDCA, carboplatin; PEM, pemetrexed; S-1, tegafur+gimeracil+oteracil; CDDP, cisplatin; LipoDOX, doxorubicin liposomal; TAS102, trifluridine; CMF, cyclophosphamide+methotrexate+5-FU; T-DM1, trastuzumab emtansine 


\begin{tabular}{|lcc|}
\hline Number of patients (male/female) & 151 & (63/88) \\
\hline S-1+DOC & 2 & $1.3 \%$ \\
\hline Other (CMF/Eribulin/T-DM1) & 3 & $2.0 \%$ \\
\hline $\begin{array}{l}\text { L-OHP, oxaliplatin; Bmab, bevacizumab; Pmab, panitumumab; Tmab, trastuzumab; PTX, paclitaxel; Nab-PTX, nanoparticle } \\
\text { albumin-bound paclitaxel; Cmab, cetuximab; GEM, gemcitabine; CPT-11, irinotecan; DOC, docetaxel; EPI, epirubicin; VNR, } \\
\begin{array}{l}\text { vinorelbine; PER, pertuzumab; FOLFIRINOX/FOLFOXIRI, L-OHP+CPT-11+5-FU; CBDCA, carboplatin; PEM, pemetrexed; S-1, } \\
\text { tegafur+gimeracil+oteracil; CDDP, cisplatin; LipoDOX, doxorubicin liposomal; TAS102, trifluridine; CMF, } \\
\text { cyclophosphamide+methotrexate+5-FU; T-DM1, trastuzumab emtansine }\end{array}\end{array}$ \\
\hline
\end{tabular}

\section{Pharmaceutical intervention for adverse events}

The pharmaceutical interventions for adverse events are shown in Table 2. The adverse event with the most interventions was nausea and vomiting (33.1\%; 50/210), followed by peripheral neuropathy $(23.8 \% ; 36 / 210)$, skin disorder $(20.5 \%$; $31 / 210)$, pain $(19.2 \% ; 29 / 210)$, and oral mucositis $(16.6 \% ; 25 / 210)$. The most common intervention for nausea and vomiting was oral administration of a D2 receptor blocker (48.0\%), followed by olanzapine (18.0\%), and aprepitant (12.0\%). For peripheral neuropathy, the most common intervention was oral administration of duloxetine (47.2\%) followed by neuropathic painalleviating agents (38.9\%), and cryotherapy (11.1\%). External application of steroid cream (38.7\%) was the most common intervention for skin disorders, followed by heparinoids (25.8\%), and oral administration of $\mathrm{H} 1$ receptor blockers (12.9\%). For pain, the most common intervention was oral administration of non-steroidal anti-inflammatory drugs (44.8\%), followed by opioids (37.9\%), and acetaminophen (24.1\%). 
Table 2

Pharmaceutical intervention for adverse events

\begin{tabular}{|c|c|c|c|}
\hline Adverse event & Intervention & Number & Rate \\
\hline \multirow{7}{*}{$\begin{array}{l}\text { Nausea and vomiting } \\
(n=50)\end{array}$} & D2 blocker & 24 & $48.0 \%$ \\
\hline & Olanzapine & 9 & $18.0 \%$ \\
\hline & Aprepitant & 6 & $12.0 \%$ \\
\hline & 5-HT3 receptor antagonist & 4 & $8.0 \%$ \\
\hline & Proton-pump inhibitor (PPI) & 3 & $6.0 \%$ \\
\hline & Mirtazapine & 2 & $4.0 \%$ \\
\hline & Others (Camostat/Butylscopolamine/Dexamethasone) & 3 & $6.0 \%$ \\
\hline \multirow{4}{*}{$\begin{array}{l}\text { Peripheral neuropathy } \\
(n=36)\end{array}$} & Duloxetine & 17 & $47.2 \%$ \\
\hline & Neuropathic pain alleviating agent & 14 & $38.9 \%$ \\
\hline & Cryotherapy & 4 & $11.1 \%$ \\
\hline & Dose reduction of oxaliplatin & 1 & $2.8 \%$ \\
\hline \multirow[t]{6}{*}{ Skin disorder $(n=31)$} & Steroid cream & 12 & $38.7 \%$ \\
\hline & Heparinoids & 8 & $25.8 \%$ \\
\hline & $\mathrm{H} 1$ receptor blocker & 4 & $12.9 \%$ \\
\hline & Antimicrobial agent & 3 & $9.7 \%$ \\
\hline & Pemirolast & 3 & $9.7 \%$ \\
\hline & Others (Posterisan ${ }^{\circledR}$ forte/Urea cream/Crotamiton) & 3 & $9.7 \%$ \\
\hline \multirow[t]{4}{*}{ Pain $(n=29)$} & Non-steroidal anti-inflammatory drugs (NSAIDs) & 13 & $44.8 \%$ \\
\hline & Opioids & 11 & $37.9 \%$ \\
\hline & Acetaminophen & 7 & $24.1 \%$ \\
\hline & Shakuyakukanzoto* & 1 & $3.4 \%$ \\
\hline \multirow[t]{4}{*}{ Oral mucositis $(n=25)$} & Sodium azulenesulfonate preparation & 15 & $60.0 \%$ \\
\hline & Steroid & 7 & $28.0 \%$ \\
\hline & Sodium alginate & 2 & $8.0 \%$ \\
\hline & Zinc preparation & 2 & $8.0 \%$ \\
\hline \multirow[t]{5}{*}{ Diarrhea $(n=15)$} & Probiotics & 9 & $60.0 \%$ \\
\hline & Loperamide & 4 & $26.7 \%$ \\
\hline & Hangeshashinto & 3 & $20.0 \%$ \\
\hline & Aluminium silicate & 2 & $13.3 \%$ \\
\hline & Others (Butylscopolamine/Alubumin tannate) & 2 & $13.3 \%$ \\
\hline Dysgeusia $(n=12)$ & Zinc preparation & 12 & $100.0 \%$ \\
\hline \multirow[t]{2}{*}{ Malaise $(n=6)$} & Tapering of dexamethasone & 5 & $83.3 \%$ \\
\hline & Hochuekkito* & 1 & $16.7 \%$ \\
\hline
\end{tabular}




\begin{tabular}{|lcc|}
\hline Adverse event $\quad$ Intervention & Number & Rate \\
\hline$*$ Shakuyakukanzoto and Hochuekkito are traditional herbal medicines commonly used in North-East Asian countries \\
\hline
\end{tabular}

\section{Relationship between pharmaceutical intervention and EQ-5D-5L utility values}

The median EQ-5D-5L utility value and proportion of patients scoring at level 2 or higher in each dimension pre- and postintervention are shown in Table 3. Overall, EQ-5D-5L utility values significantly improved ( 0.8197 pre to 0.8603 post; $P<0.01$ ) after pharmaceutical intervention for adverse events. A significant change in the proportion of patients with level 2 or higher scores pre- and post-intervention was observed only in the 'mobility' dimension ( $37.1 \%$ pre to $30.5 \%$ post; $P=0.014$ ).

Table 3

Comparison of EuroQol 5 Dimension-5 Level (EQ-5D-5L) utility values between pre-and post-intervention.

\begin{tabular}{|llll|}
\hline & Pre-intervention & Post-intervention & Pvalue \\
\hline Utility value (IQR) & $0.8197(0.7096-0.8978)$ & $0.8603(0.7543-0.9384)$ & $<0.01$ \\
\hline Dimension & Proportion of level $\geq 2(\%)$ & \\
\hline Mobility & 37.1 & 30.5 & 0.014 \\
\hline Personal care & 14.3 & 13.3 & 0.804 \\
\hline Usual activities & 41.9 & 38.1 & 0.185 \\
\hline Pain/discomfort & 65.2 & 58.6 & 0.071 \\
\hline Anxiety/depression & 45.7 & 41.0 & 0.144 \\
\hline
\end{tabular}

\section{Pre- and post-intervention changes in EQ-5D-5L utility values and incidence of grade 2 or higher adverse events}

Figure 2 shows the median EQ-5D-5L utility values pre- and post-intervention for each adverse event. EQ-5D-5L utility values significantly improved from pre- to post-intervention for nausea and vomiting ( 0.8145 pre to 0.8603 post; $P=0.016)$, peripheral neuropathy ( 0.7798 pre to 0.7988 post; $P=0.032)$, and pain $(0.7625$ pre to 0.8197 post; $P=0.035)$. Although not statistically significant, the median EQ-5D-5L utility values tended to increase for skin disorder ( 0.8603 pre to 0.8978 post; $P=0.057)$, oral mucositis ( 0.8603 pre to 0.8978 post; $\mathrm{P}=0.121)$ and diarrhea $(0.7923$ pre to 0.8197 post; $P=0.328)$. Comparing the proportion of level 2 or higher scores in the 5 dimensions for each adverse event, there were no significant changes pre- and postintervention (data not shown).

The incidence of adverse events is shown in Figure 3. The incidence of grade 2 or higher nausea and vomiting (56.0\% pre to $40.0 \%$ post; $P=0.18)$, skin disorder (22.6\% pre to $9.7 \%$ post; $P=0.125)$, pain (31.0\% pre to $20.7 \%$ post; $P=1.0)$, oral mucositis (36.0\% pre to $16.0 \%$ post; $P=0.25$ ), diarrhea (33.4\% pre to $20.0 \%$ post; $P=1.0$ ) and dysgeusia (58.3\% pre to $33.3 \%$ post; $P$ $=0.375$ ) tended to be lower post-intervention, albeit that these differences were not significant.

\section{Discussion}

In this study, we investigated the effects of pharmaceutical interventions for adverse events provided by pharmacists in collaboration with physicians on QOL in patients receiving outpatient cancer chemotherapy. Evaluation by EQ-5D-5L revealed that interventions were associated with a significant post-intervention improvement in patient QOL. In particular, interventions for nausea and vomiting, pain, and peripheral neuropathy were associated with significant improvements in QOL before and after the intervention. These results indicate that pharmaceutical intervention provided by pharmacists in collaboration with physicians for adverse events is useful for improving QOL in cancer chemotherapy. 
Comparison of EQ-5D-5L utility values before and after pharmaceutical intervention for each of the 5 dimensions revealed that mobility was significantly improved post-intervention. The proportion of patients with a mobility level higher than 2 significantly decreased after pharmaceutical intervention. Similarly, a population-based cohort study of women diagnosed with breast cancer reported that patients with higher exercise metabolic equivalent scores had higher total QOL scores [23]. Eyl et al. also reported that QOL in long-term colorectal cancer survivors was higher in those who were more physically active than non-active survivors [24]. These findings indicate that improved mobility after the receipt of pharmaceutical intervention may contributed to enhancing QOL.

EQ-5D-5L utility values significantly increased after pharmaceutical intervention for nausea and vomiting. Hagiwara et al. showed that grade 1 nausea negatively impacted patient QOL [25]. Chemotherapy-induced nausea and vomiting are common side effects that can greatly impact patients receiving outpatient cancer-chemotherapy [26]. Additionally, the Clinical Practice Guidelines for Antiemesis from the Japan Society of Clinical Oncology highlight the importance of managing delayed nausea and vomiting during outpatient cancer-chemotherapy [27]. At Gifu University Hospital, pharmacists work in collaboration with physicians to select antiemetic agents in consideration of comorbidities and the appearance of nausea and vomiting after chemotherapy. Andrea et al. reported that antiemetic responses and patient satisfaction were significantly improved with the use of supportive medication prescribed according to evidence-based treatment guidelines and patient counseling for the management of treatment-associated adverse events [28]. Thus, individualized antiemetic treatment may contribute to improving patient QOL. Moreover, we found that EQ-5D-5L utility values were significantly improved when aprepitant was orally administered to treat nausea and vomiting (data not shown). Similarly, Thomas et al. reported that the addition of aprepitant to a standard regimen resulted in significantly less chemotherapy-induced nausea and vomiting and improved QOL [29].

Pharmaceutical interventions for pain also significantly improved EQ-5D-5L values. In particular, post-intervention EQ-5D-5L utility values significantly improved after opioids were added to the pain treatment regimen (data not shown). This finding is consistent with previous reports that QOL was improved after oral administration of opioids for severe pain in patients with cancer [30]. Clinical guidelines for cancer pain management suggest that opioids should be administered to cancer patients with moderate-to-severe pain [31-34]. The relationship between QOL and pain is inversely proportional in patients with breast cancer [35]. Therefore, the oral administration of opioids can relieve more severe pain and significantly improved patient QOL.

EQ-5D-5L utility values were also significantly improved after pharmaceutical intervention for peripheral neuropathy. Administration of pregabalin and duloxetine [36,37] and the use of frozen gloves (cryotherapy) [38] are reported to improve QOL in patients with peripheral neuropathy. Although overall EQ-5D-5L utility values improved post-intervention, no significant differences were observed in the incidence of grade 2 or higher adverse events. According to the CTCAE, grade 2 peripheral neuropathy is a symptom that affects daily life, and the range of symptoms included in this classification is wide. In terms of the CTCAE assessment of peripheral neuropathy, pharmaceutical interventions were found to improve symptoms, which may have contributed to enhancing patient QOL. To assess the effect of pharmaceutical intervention for peripheral neuropathy, we considered that it would be necessary to use not only CTCAE but also NRS; however, we did not assess peripheral neuropathy using NRS or VAS in this study, and determining which scale is more appropriate for assessment of peripheral neuropathy is a question for future research.

In this study, we demonstrated that pharmaceutical intervention provided by pharmacists in collaboration with physicians contributed to QOL enhancement. Comprehensive pharmaceutical care increases patients' chemotherapy-related knowledge, improves positive emotions, facilitates the management of chemotherapy-induced adverse events, and improves QOL [39]. An association between lower QOL and increased all-cause mortality has been reported [40]. Accordingly, to improve therapeutic effects, QOL should be maintained or enhanced in patients undergoing outpatient chemotherapy. The relationship between QOL enhancement and therapeutic effects warrants elucidation in future study.

Several limitations of this study should be acknowledged. First, because we were unable to compare our results with a control group that did not receive pharmaceutical intervention, it is unclear whether the improvement in QOL was due to pharmaceutical intervention or time. Second, the degree of cancer progression and the environment surrounding patients receiving outpatientcancer chemotherapy can strongly impact QOL. We did not give sufficient consideration to patient condition, such as tumor

Page 10/16 
metastasis, line of treatment, employment, and marital status. Furthermore, this study was a single-center study with a small sample size, which could result in exposure of the study population to a certain common bias. Further studies with a larger sample size are needed to verify our findings.

\section{Conclusion}

This study suggests that the enhancement of supportive care for adverse events, such as nausea and vomiting, peripheral neuropathy, and pain, may improve the QOL of outpatients undergoing chemotherapy. Pharmaceutical intervention provided by pharmacists in collaboration with physicians is useful in maintaining and improving QOL and in the management of adverse events during cancer treatment.

\section{Declarations}

\section{ACKNOWLEDGMENTS}

We appreciate the participants for their kind contribution to this study.

\section{Funding}

This study did not receive funding from any funding source.

\section{Conflicts of interest/Competing interests}

K. Yoshida has received honoraria for lectures from Chugai Pharmaceutical Co., Ltd., Taiho Pharmaceutical Co., Ltd., Takeda Pharmaceutical Co., Ltd., Eli Lilly and Company, Yakult Honsha Co., Ltd., Merck Sharp \& Dohme Co., Ltd., Daiichi Sankyo Co., Ltd., Ono Pharmaceutical Co., Ltd., Merck Serono Co., Ltd., Johnson \& Johnson Co., Ltd., Covidien Co., Ltd., Eisai Co., Ltd., Otsuka Pharmaceutical Co., Ltd., Sanofi K.K., Nippon Kayaku Co., Ltd., Asahi Kasei Co., Ltd., Tsumura Co., Ltd., EA Pharma Co., Ltd., Bayer Yakuhin Co., Ltd., Olympus Co., Ltd., Terumo Co., Ltd., Bristol-Myers Squibb Co., Ltd., Denka Co., Ltd., Teijin Co., Ltd., SBI Pharmaceuticals Co., Ltd., Intuitive Surgical Co., Ltd., Novartis Pharma K.K., and Pfizer Inc.; and research funding from Chugai Pharmaceutical Co., Ltd., Taiho Pharmaceutical Co., Ltd., Takeda Pharmaceutical Co., Ltd., Eli Lilly and Company, Yakult Honsha Co., Ltd., Merck Sharp \& Dohme Co., Ltd., Daiichi Sankyo Co., Ltd., Ono Pharmaceutical Co., Ltd., Merck Serono Co., Ltd., Johnson \& Johnson Co., Ltd., Covidien Co., Ltd., Eisai Co., Ltd., Otsuka Pharmaceutical Co., Ltd., Sanofi K.K., Nippon Kayaku Co., Ltd., Asahi Kasei Co., Ltd., Tsumura Co., Ltd., Kyowa Hakko Kirin Co., Ltd., Astellas Pharma Co., Ltd., Toyama Chemical Co., Ltd., Kinetic Concepts Co., Ltd., Abbott Japan Co., Ltd., and Toray Industries, Co., Ltd. outside the submitted work.

T. Takahashi has received honoraria for lectures from Takeda Pharmaceutical Co., Ltd.

A. Makiyama has received honoraria for lectures from Eli Lilly and Company, Taiho Pharmaceutical Co., Ltd. and Takeda Pharmaceutical Co., Ltd.

The other authors have no conflicts of interest.

\section{Consent to participate/Consent for publication}

Informed consent was not obtained because this was a retrospective observational study. We posted information (opt-out enrollment method) about this study on the website of the hospital.

\section{Ehics approval}

This retrospective study involving human participants was in accordance with the ethical standards of the institutional and national research committee and with the 1964 Helsinki Declaration and its later amendments or comparable ethical standards. Ethical approval for the study was obtained from the ethics committees of Gifu Pharmaceutical University (Approval Number: 39) and Gifu University Graduate School of Medicine (Approval number: 2021-03). 
N/A

\section{Code availability}

N/A

Authors' contributions

Conceptualization, H.F.; methodology, H.F.; formal analysis, H.F. and Y.U.; investigation, H.F., Y.U.; data curation, C.H., K.O., K.S., M.K., S.S., S.Y., D.W., H.K.-H., H.I., and M.K.; writing-original draft preparation, H.F. and Y.U.; Writing-review and editing, H.F., Y.U., H.I., R.K., N.M., T.T., A.M., K.Y., H.H. and A.S.; supervision, A.S. All authors have read and agreed to the published version of the manuscript.

\section{References}

1. World Health Organization (2021) Cancer. https://www.who.int/en/news-room/fact-sheets/detail/cancer, 13 October accessed.

2. Torre LA, Siegel RL, Ward EM et al (2016) Global Cancer Incidence and Mortality Rates and Trends — An Update. Cancer Epidemiol Biomarkers Prev 25 (1) :16-27. https://doi.org/10.1158/1055-9965.EPI-15-0578

3. Gradishar WJ, Tjulandin S, Davidson N et al (2005) Phase III trial of nanoparticle albumin-bound paclitaxel compared with polyethylated castor oil-based paclitaxel in women with breast cancer. J Clin Oncol 23(31):7794-7803.

http://doi.org/10.1200/JC0.2005.04.937

4. Saito M, Aogi K, Sekine I et al (2009) Palonosetron plus dexamethasone versus granisetron plus dexamethasone for prevention of nausea and vomiting during chemotherapy: a double-blind, double-dummy, randomised, comparative phase III trial. Lancet Oncol 10(2):115-124. https://doi.org/10.1016/S1470-2045(08)70313-9

5. Ishiura Y, Terasaki Y, Yamamoto H et al. (2007) Analysis of factors associated with quality of life for patients with nonsmall lung cancer receiving outpatient vinorelbine therapy as alternative inpatient therapy. Gan To Kagaku Ryoho 34(9):1401-4.

6. Miyake T, Hashida T, Kobayashi M et al (2008) Survey on Use of Amrubicin Hydrochloride in Outpatients with Lung Cancer and Evaluation of its Hematologic Toxicity. Japanese Journal of Pharmaceutical Health Care and Sciences 34, 268-273.

7. Tsai SC, Liu LN, Tang ST et al (2010) Cancer pain as the presenting problem in emergency departments: incidence and related factors. Support Care Cancer 18(1):57-65. https://doi.org/10.1007/s00520-009-0630-6

8. Hirose $\mathrm{C}$, Fujii $\mathrm{H}$, lihara $\mathrm{H}$ et al (2020) Real-world data of the association between quality of life using the EuroQol 5 Dimension 5 Level utility value and adverse events for outpatient cancer chemotherapy. Support Care Cancer 28(12):59435952. https://doi.org/10.1007/s00520-020-05443-8

9. Prieto-Callejero B, Rivera F, Fagundo-Rivera J et al (2020) Relationship between chemotherapy-induced adverse reactions and health-related quality of life in patients with breast cancer. Medicine (Baltimore). 14;99(33):e21695.

https://journals.Iww.com/mdjournal/Fulltext/2020/08140/Relationship_between_chemotherapy_induced_adverse.76.aspx. Accessed 13 October 2021

10. Iwamoto S, Ooki A, Morita S et al (2018) A prospective Phase II study to examine the relationship between quality of life and adverse events of first-line chemotherapy plus cetuximab in patients with KRAS wild-type unresectable metastatic colorectal cancer: QUACK trial. Cancer Med 7(9):4217-4227. https://doi.org/10.1002/cam4.1623

11. de Mol M, Visser S, den Oudsten BL et al (2019) Frequency of low-grade adverse events and quality of life during chemotherapy determine patients' judgement about treatment in advanced-stage thoracic cancer. Support Care Cancer 27(9):3563-3572 https://doi.org/10.1007/s00520-019-4659-x

Page 12/16 
12. Mae Y, Yokokawa T, Kawakami K et al (2011) Usefulness of Pharmaceutical Outpatient Clinic in XELOX Therapy. Japanese Journal of Pharmaceutical Health Care and Science 37(11):611-615.

13. Sudou M, Morii H, Sakanaka M et al (2013) Introduction of pharmaceutical care and its effects in outpatients with cancer chemotherapy. Japanese Journal of Pharmaceutical Health Care and Science 39(2):77-84.

14. Colombo LRP, Aguiar PM, Lima TM et al (2017) The effects of pharmacist interventions on adult outpatients with cancer: $A$ systematic review. J Clin Pharm Ther 42(4):414-424. https://doi.org/10.1111/jcpt.12562

15. Tanaka K, Hori A, Tachi T et al (2018) Impact of pharmacist counseling on reducing instances of adverse events that can affect the quality of life of chemotherapy outpatients with breast Cancer. J Pharm Health Care Sci 4: 9. https://doi.org/10.1186/s40780-018-0105-3

16. Farrag Dina K, Sabri Nagwa A, Tawfik Amr S et al (2020) Evaluation of the clinical effect of pharmacist intervention, European Journal of Oncology Pharmacy. Jan-Mar; 3(1).

https://journals.Iww.com/ejop/Fulltext/2020/03000/Evaluation_of_the_clinical_effect_of_pharmacist.3.aspx. Accessed 13 October 2021.

17. Periasamy U, Mohd-Sidik S, Akhtari-Zavare M et al (2020) Effects of Counselling on Quality of Life among Cancer Patients in Malaysia: A Randomized Controlled Trial. Iran J Public Health 49(10):1902-1911.

18. Herdman M, Gudex C, Lloyd A et al (2011) Development and preliminary testing of the new five-level version of EQ-5D (EQ5D-5L). Qual Life Res. 20(10):1727-1736. https://doi.org/10.1007/s11136-011-9903-x

19. Brauer CA, Rosen AB, Greenberg D et al (2006) Trends in the measurement of health utilities in published cost-utility analyses. Value Health 9(4):213-8 https://doi.org/10.1111/j.1524-4733.2006.00116.x

20. Ikeda S, Shiroiwa T, Igarashi et al (2015) A. Developing a Japanese version of the EQ-5D-5L value set. J. Natl. Inst. Public Health 64: 47-55.

21. Okamoto T, Hashimoto K, Ohashi M et al (2004) Survey on health-related quality of life (HRQOL) and cost-effectiveness for rehabilitation hospital inpatients by EuroQOL. The Japanese Journal of Rehabilitation Medcine 41(10):678-685.

22. US Department of Health and Human Services (2009) National Institutes of Health National Cancer Institute Common terminology criteria for adverse events (CTCAE) version 4.0. Available: https://www.eortc.be/services/doc/ctc/. Accessed 15

23. Chen $X$, Zheng Y, Zheng W et al (2009) The effect of regular exercise on quality of life among breast cancer survivors. Am $J$ Epidemiol 1;170(7):854-862. https://doi.org/10.1093/aje/kwp209

24. Eyl RE, Xie K, Koch-Gallenkamp L et al (2018) Quality of life and physical activity in long-term ( $\geq 5$ years post-diagnosis) colorectal cancer survivors - systematic review. Health Qual Life Outcomes 16(1):112. https://doi.org/10.1186/s12955-0180934-7

25. Hagiwara Y, Shiroiwa T, Shimozuma K et al (2018) Impact of Adverse Events on Health Utility and Health-Related Quality of Life in Patients Receiving First-Line Chemotherapy for Metastatic Breast Cancer: Results from the SELECT BC Study. Pharmacoeconomics 36(2):215-223. https://doi.org/10.1007/s40273-017-0580-7

26. Ihbe-Heffinger A, Ehlken B, Bernard R et al (2004) The impact of delayed chemotherapy-induced nausea and vomiting on patients, health resource utilization and costs in German cancer centers. Ann Oncol 15(3):526-536.

https://doi.org/10.1093/annonc/mdh110

27. Aogi K, Takeuchi H, Saeki T et al (2021) Optimizing antiemetic treatment for chemotherapy-induced nausea and vomiting in Japan: Update summary of the 2015 Japan Society of Clinical Oncology Clinical Practice Guidelines for Antiemesis. Int J Clin Oncol. 26:1-17. https://doi.org/10.1007/s10147-020-01818-3

28. Liekweg A, Westfeld M, Braun M et al (2012) Pharmaceutical care for patients with breast and ovarian cancer. Support Care Cancer. 20(11):2669-2677. https://doi.org/10.1007/s00520-012-1385-z

29. Schmitt T, Goldschmidt H, Neben K et al (2014) Aprepitant, granisetron, and dexamethasone for prevention of chemotherapy-induced nausea and vomiting after high-dose melphalan in autologous transplantation for multiple myeloma: results of a randomized, placebo-controlled phase III trial. J Clin Oncol. 20;32(30):3413-3420.

https://doi.org/10.1200/JCO.2013.55.0095

Page 13/16 
30. Leppert W, Nosek K (2019) Comparison of the Quality of Life of Cancer Patients with Pain Treated with Oral ControlledRelease Morphine and Oxycodone and Transdermal Buprenorphine and Fentanyl. Curr Pharm Des. 25(30):3216-3224. https://doi.org/10.2174/1381612825666190717091230

31. Marinangeli F, Ciccozzi A, Leonardis M et al (2004) Use of strong opioids in advanced cancer pain: a randomized trial. J Pain Symptom Manage 27(5):409-416. https://doi.org/10.1016/j.jpainsymman.2003.10.006

32. Maltoni M, Scarpi E, Modonesi C et al (2005) A validation study of the WHO analgesic ladder: a two-step vs three-step strategy. Support Care Cancer. 13(11):888-894. https://doi.org/10.1007/s00520-005-0807-6

33. Bandieri E, Romero M, Ripamonti Cl et al (2016) Randomized Trial of Low-Dose Morphine Versus Weak Opioids in Moderate Cancer Pain. J Clin Oncol. 34(5):436-442. https://doi.org/10.1200/JC0.2015.61.0733

34. Portenoy RK (2011) Treatment of cancer pain. Lancet. 2011;377(9784):2236-2247. https://doi.org/10.1016/S01406736(11)60236-5

35. Costa WA, Monteiro MN, Queiroz JF et al (2017) Pain and quality of life in breast cancer patients. Clinics (Sao Paulo) 72(12):758-763. https://doi.org/10.6061/clinics/2017(12)07

36. Avan R, Janbabaei G, Hendouei $\mathrm{N}$ et al (2018) The effect of pregabalin and duloxetine treatment on quality of life of breast cancer patients with taxane-induced sensory neuropathy: A randomized clinical trial. J Res Med Sci 6;23:52. https://doi.org/10.4103/jrms.JRMS_1068_17

37. Smith EM, Pang H, Cirrincione $\mathrm{C}$ et al (2013) Effect of duloxetine on pain, function, and quality of life among patients with chemotherapy-induced painful peripheral neuropathy: a randomized clinical trial. JAMA 309(13):1359-1367. https://doi.org/10.1001/jama.2013.2813

38. Beijers AJM, Bonhof CS, Mols F et al (2020) Multicenter randomized controlled trial to evaluate the efficacy and tolerability of frozen gloves for the prevention of chemotherapy-induced peripheral neuropathy. Ann Oncol 31(1):131-136. https://doi.org/10.1016/j.annonc.2019.09.006

39. Wang Y, Wu H, Xu F (2015) Impact of Clinical Pharmacy Services on KAP and QOL in Cancer Patients: A Single-Center Experience. Biomed Res Int. 2015; 2015: 502431. https://doi.org/10.1155/2015/502431

40. Ratjen I, Schafmayer C, Enderle J et al (2018) Health-related quality of life in long-term survivors of colorectal cancer and its association with all-cause mortality: a German cohort study. BMC Cancer 18(1):1156. https://doi.org/10.1186/s12885018-5075-1

\section{Figures}


Among cases with intervention for adverse events during outpatient cancer chemotherapy,

302 answered the EQ-5D-5L

Adverse events worsened except for intervention items: 84 Incomplete QOL data: 8

210 cases were finally analyzed

Figure 1

CONSORT diagram

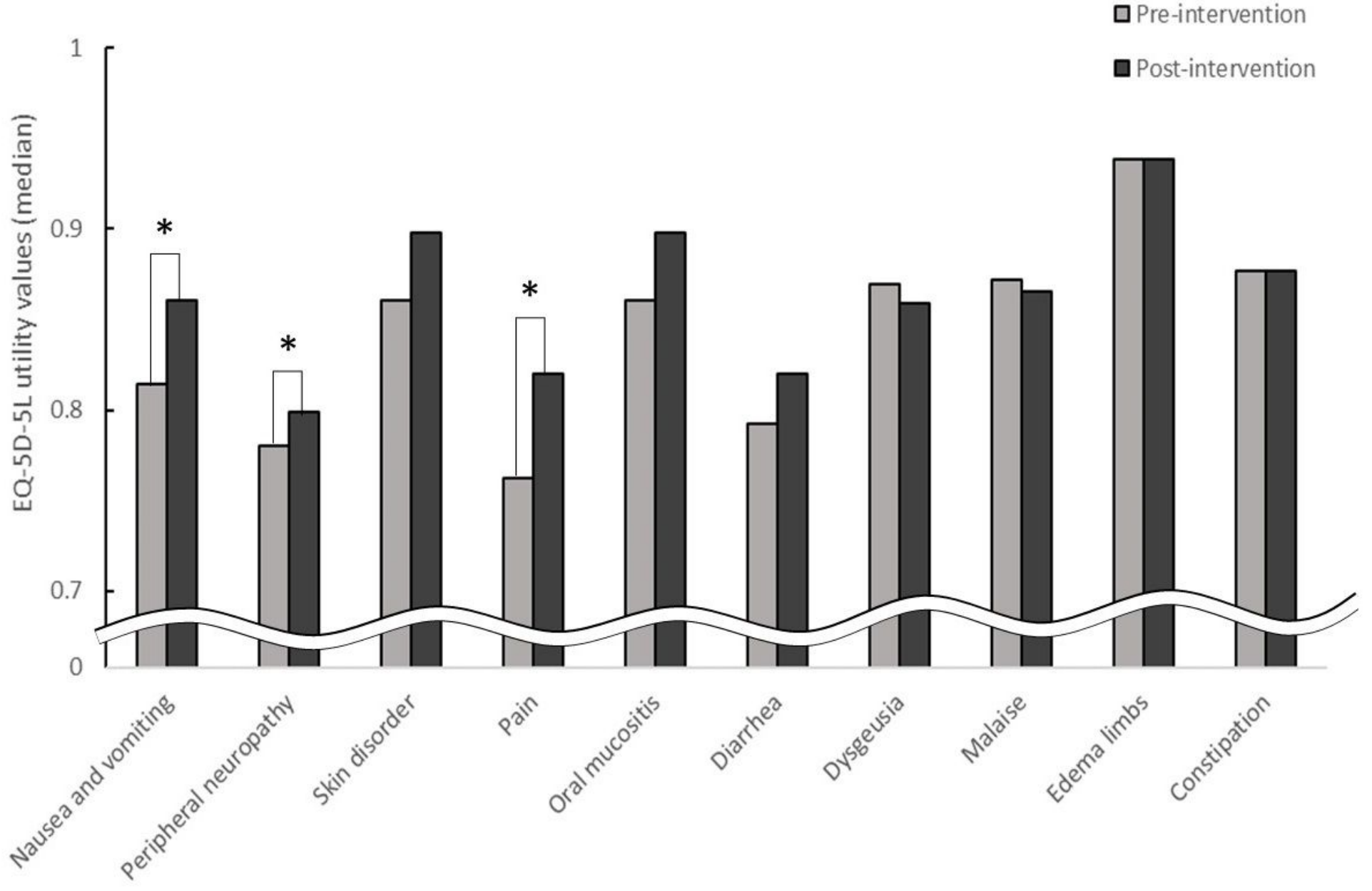

Figure 2 
Comparison of median EQ-5D-5L utility values pre- and post-intervention for each adverse event The Wilcoxon signed-rank test was used to assess differences $\left({ }^{*} \mathrm{P}<0.05\right)$. Pre-intervention, adverse event onset; post-intervention, after pharmaceutical intervention.

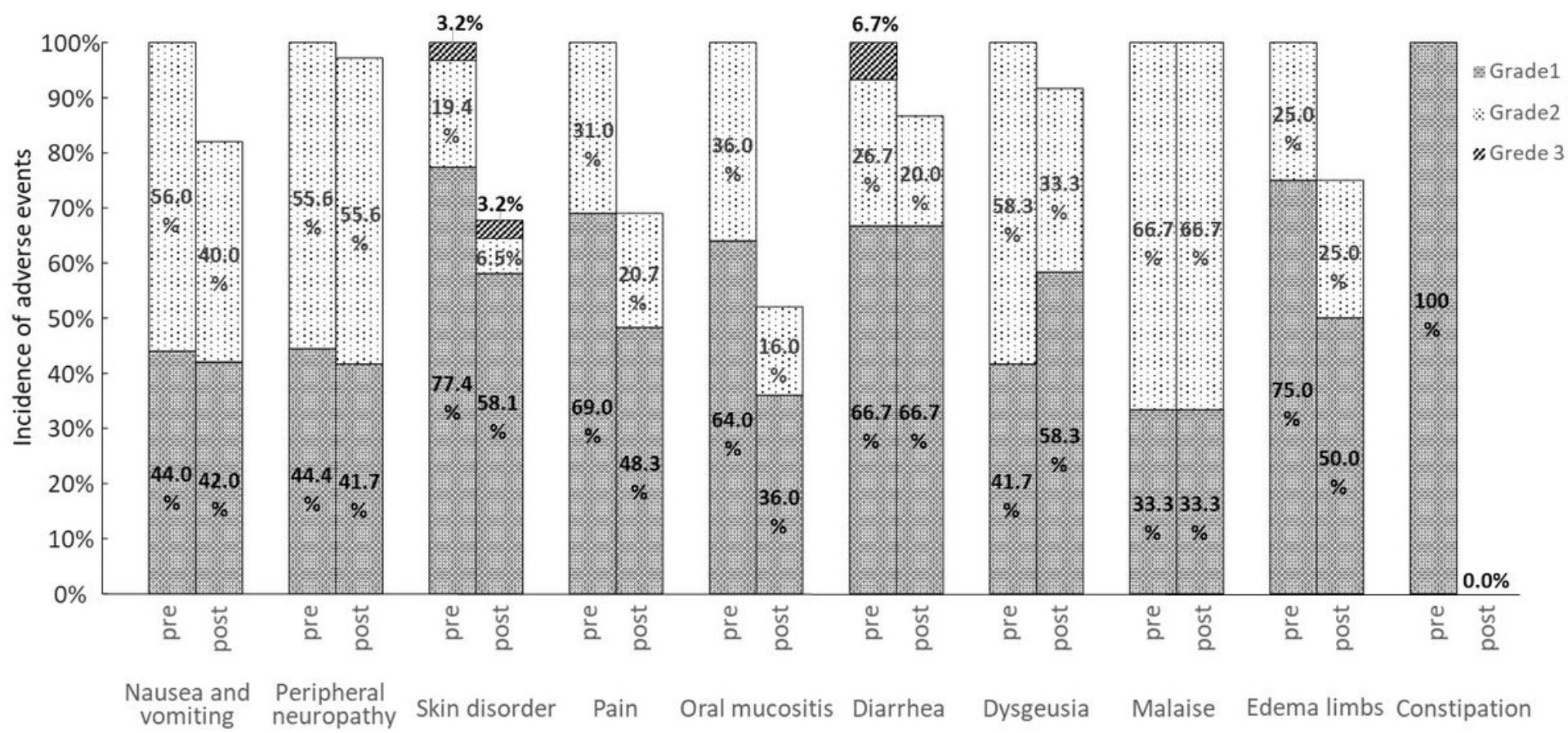

\section{Figure 3}

Comparison of adverse event incidence and grade pre- and post-intervention McNemar's test was used to compare the incidence of grade 2 or higher adverse events before and after pharmaceutical intervention 